\title{
Is It Time to Review our Educational Program for the Electrocardiography Operators?
}

\author{
Habibollah Saadat ${ }^{1}$, Hossein Tajdini ${ }^{2}$, Zahra Saadat ${ }^{1}$, Vahid \\ Eslami ${ }^{1}$, Mehdi Sheibani ${ }^{1}$,Babak Sharifkashani ${ }^{1}$,Roxana \\ Sadeghi ${ }^{1}$, Isa Khaheshi ${ }^{1, *}$ \\ ${ }^{1}$ Cardiovascular Research Center, Shahid Beheshti University of Medical Sciences, \\ Tehran, Iran \\ ${ }^{2}$ Stopford Building, University of Manchester, Manchester, United Kingdom \\ * Corresponding author: Isa Khaheshi, Cardiovascular Research Center, Shahid Be-

heshti University of Medical Sciences, Tehran, Iran.E-mail:isa_khaheshi@yahoo.com

DOI: $10.21859 / \mathrm{ijcp}-020302$

Submited: 04.10.2017

Accepted: 05.17.2017

Keywords:

Electrocardiography

Electrodes, Misplacement

Leads

(C) 2017. International Journal of

Cardiovascular Practice.

Electrocardiography (ECG) is one of the imperative diagnostic tools in cardiology. In spite of considerable advances in imaging and diagnostic methods in cardiology, ECG has still maintained its crucial role in the diagnosis of variant problems and pathologies; moreover, it would be so helpful in guiding rapid and appropriate therapies in the field of cardiology and internal medicine [1-4]. Accordingly, performance of correct and standard ECG is an inevitable need for each health system; missing the probable errors in ECG and inability to correct them may lead to unfavorable outcomes including misdiagnosis and inappropriate treatment [4-7]. This pilot study was conducted to evaluate the familiarity of ECG operators with essential points of ECG-performance.

In the current pilot study, ECG operators were interviewed by an educated student. All of the questions were similar to each operator and they were asked subjective questions, together with objective ones. The questions were asked according to a standard questionnaire. A student of biomedical sciences was well-educated to ask questions, without any possible guiding clues. This descriptive pilot study was conducted from June 2016 to November 2016. In this study, 150 ECG operators including 103 females $(68.7 \%)$ and 47 males $(31.3 \%)$ were interviewed. The mean age of them was $30.8 \pm 10.5$ years. Also, 55(36.7\%), 21(14\%), 67(44.7\%), $6(4 \%)$ and $1(0.7 \%)$ of them had high school diploma, Associate, Bachelor, Master and PHD degrees, respectively.

Only $47.3 \%$ of them plugged off the ECG device when they performed a standard ECG. Plugging off the device during ECG-performance perhaps seems to be a simple and trivial issue; however, more than half of the ECG-operators performed ECG while the device was plugged in, that may lead to some disturbance, including noises and misinterpretation.
On the other hand, $72.7 \%$ of them plugged in the ECG device at the end of the usage of the ECG-device; once, they were asked to show speed and voltage buttons, $74.7 \%$ and $72 \%$ of them made true answers. As objective questions, ECG with changed speed and voltage was presented to them to recognize the speed or voltage errors; only $47.3 \%$ and $46 \%$ of them recognize the speed and voltage errors, respectively. In this study, $65.3 \%$ of the subjects controlled and checked the set-up of the ECG device including speed and voltage before performing ECG and $4 \%$ at the beginning of working shift; however, about $30.7 \%$ did not check the ECG device in view of speed and voltage set-up. Also, $96.7 \%$ and $89.3 \%$ of the ECG operators defined correct places of the limb and chest leads, respectively. When they were asked to perform a classic left sided-ECG, $96.7 \%$ and $96 \%$ of them showed correct performance of installation of limb and chest leads, respectively. When they were asked to perform right sided-ECG, $71.3 \%$ showed true performance. The true performance was lower when they were asked to perform posterior sided-ECG, in which, only $49.3 \%$ revealed true performance. Once, they were asked to perform Lewis-ECG leads, the number of true answers was dramatically low, in which, only 8.7\% expressed the correct performance. When an ECG with noise errors was presented to them, $88 \%$ of them could recognize the noise problem in the ECG. As an interesting point, all of them suggested at least one true solution to eliminate or diminish noises in ECG. As our pilot study showed, there were several pitfalls in performance of a standard ECG and some of them should be considered carefully. Although our study was a pilot study with a limited sample population, it was clear that most of the operators performed only routine practices and were not familiar with special issues on elec- 
trocardiography. In conclusion, performance of a correct and standard ECG is an inevitable need. It seems that there are numbers of pitfalls in the knowledge of ECG-operators. Careful re-evaluation of educational programs and settling intermittent workshops are warranted.

\section{CONFLICTS OF INTERESTS}

There is no conflict of interest.

\section{REFERENCES}

1. Bays de Luna A. Basic Electrocardiography: Normal and Abnormal ECG Patterns. Malden, Massachusetts: Wiley-Blackwell; 2007.

2. Goldberger AL. Giles f. Filley lecture. Complex systems. Proc Am Thorac Soc. 2006;3(6):467-71. DOI: 10.1513/pats.200603-028MS
PMID: 16921107

3. Baltazar R. Basic and Bedside Electrocardiography. 1st ed. Philadelphia, PA: Lippincott Williams \& Wilkins; 2009.

4. Rajaganeshan R, Ludlam CL, Francis DP, Parasramka SV, Sutton R. Accuracy in ECG lead placement among technicians, nurses, general physicians and cardiologists. Int J Clin Pract. 2008;62(1):65-70. DOI: $10.1111 /$ j.1742-1241.2007.01390..x PMID: 17764456

5. Schijvenaars BJ, Kors JA, van Herpen G, Kornreich F, van Bemmel $\mathrm{JH}$. Effect of electrode positioning on ECG interpretation by computer. J Electrocardiol. 1997;30(3):247-56. DOI: 10.1016/s00220736(97)80010-6 PMID: 9261733

6. Edhouse J, Thakur RK, Khalil JM. ABC of clinical electrocardiography. Conditions affecting the left side of the heart. BMJ. 2002;324(7348):1264-7. PMID: 12028984

7. Breen C, Bond R, Finlay D. A clinical decision support tool to assist with the interpretation of the 12-lead electrocardiogram. Health Informatics J. 2017:1460458216683534. DOI: $\underline{10.1177 / 1460458216683534} \quad$ PMID: 28056601 